\title{
Realization of robust photonic crystal waveguides designed to reduce out-of-plane scattering
}

\author{
Arentoft, Jesper; Kristensen, Martin; Søndergaard, Thomas; Boltasseva, Alexandra
}

Published in:

Proceedings on 27th European Conference on Optical Communication

Link to article, DOI:

10.1109/ECOC.2001.989122

Publication date:

2001

Document Version

Publisher's PDF, also known as Version of record

Link back to DTU Orbit

Citation (APA):

Arentoft, J., Kristensen, M., Søndergaard, T., \& Boltasseva, A. (2001). Realization of robust photonic crystal waveguides designed to reduce out-of-plane scattering. In Proceedings on 27th European Conference on Optical Communication (Vol. 4, pp. 592-593) https://doi.org/10.1109/ECOC.2001.989122

\section{General rights}

Copyright and moral rights for the publications made accessible in the public portal are retained by the authors and/or other copyright owners and it is a condition of accessing publications that users recognise and abide by the legal requirements associated with these rights.

- Users may download and print one copy of any publication from the public portal for the purpose of private study or research.

- You may not further distribute the material or use it for any profit-making activity or commercial gain

- You may freely distribute the URL identifying the publication in the public portal 


\title{
Realization of robust photonic crystal waveguides designed to reduce out-of-plane scattering
}

\author{
Jesper Arentoft (1), Martin Kristensen (2), Thomas Søndergaard (3) \\ and Alexandra Boltasseva (4) \\ COM, Technical University of Denmark, Bldg. 345 west, DK-2800 Kgs. Lyngby, Denmark
} (1) ja@com.dtu.dk, (2) mk@com.dtu.dk, (3) ts@com.dtu.dk, (4) aeb@com.dtu.dk

\begin{abstract}
We have realized envirommentally stable silicon-on-insulator based photonic crystal waveguides. The waveguide structure is designed to minimize scattering at semiconductor/hole interfaces. Transmission measurements and IR pictures indicate efficient guiding through straight and bended waveguides.
\end{abstract}

\section{Introduction}

Photonic crystal $(\mathrm{PhC})$ structures that guide light by the photonic bandgap (PBG) effect are promising building blocks for miniature photonic integrated circuits $/ 1 /$. Waveguides with PBG confinement in three dimensions are preferable but difficult to fabricate and not easy to integrate on planar photonic chips. Therefore, much attention is paid to $2 \mathrm{D}$ PhCs, which confine light by the PBG effect in the plane and by total internal reflection in the out-of-plane dimension $/ 2,3 /$. One of the main challenges to overcome for $2 \mathrm{D} \mathrm{PhC}$ waveguides is random scattering at imperfect interfaces between high and low refractive index media $/ 4 /$. For a finite-height $2 \mathrm{D} \mathrm{PhC}$ structure consisting of a semiconductor slab with air holes, scattering takes place at the horizontal planar interfaces and at vertical interfaces inside the air holes. In-plane scattering losses are suppressed by the photonic crystal lattice; however, total internal reflection only occurs for light scattered out-ofplane at a shallow angle. It is therefore desirable to reduce scattering at the interfaces, either by reducing the interface roughness or by reducing the index contrast.

Successful demonstration of $\mathrm{PhC}$ waveguides with low outof-plane scattering will therefore be an important step towards large-scale integrated optical circuits. Measurement of the optical properties of $\mathrm{PhC}$ waveguides are complicated by their inherent small size. $\mathrm{A} \mathrm{PhC}$ waveguide typically has cross-sectional dimensions of $500 \mathrm{~nm}$ by $300 \mathrm{~nm}$ and length of only a few hundred $\mu \mathrm{m}$. This makes the waveguides hard to access with standard optical equipment for e.g. transmission studies.

Here we present silicon-on-insulator (SOI) based $\mathrm{PhC}$ waveguides, designed to be robust, to reduce out-of-plane scattering and to make transmission measurements possible.

\section{Design}

Our PhC structures consist of silica/silicon/silica trilayers with air holes (arranged on a triangular lattice) extending through all three layers. These structures are significantly more environmentally stable than an air-cladding version. An example of a fabricated structure is shown in Fig.1.

Our choice of hole radius, $R$, and hole spacing, $\Lambda$, is based on 2D Plane-Wave-Expansion calculations of in-plane photonic bandgaps for the structure. Fig. 2 shows the inplane photonic bandgaps for TE (in-plane) polarized light as a function of the hole radius, $R$, for infinite height photonic crystals with holes arranged on a triangular lattice in silicon. Calculated bandgaps are shown for three different structures, the difference being the media present in the holes. The solid curve corresponds to air holes, the

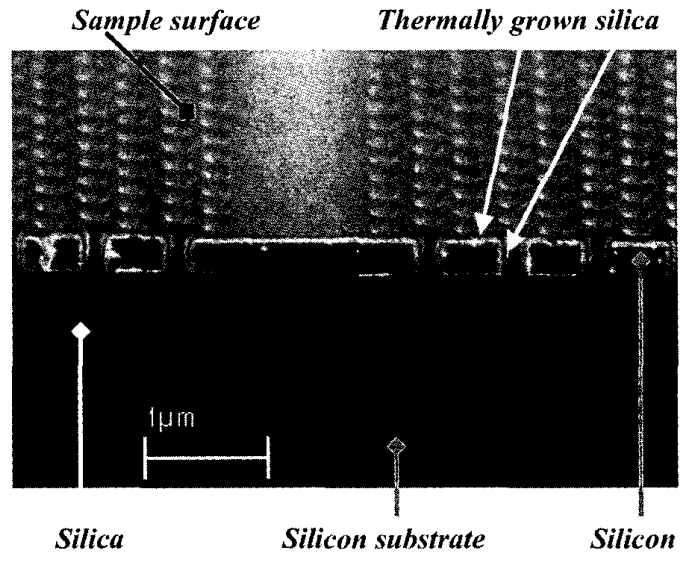

Figure 1 : SEM micrograph showing a cleaved facet of a fabricated photonic crystal waveguide structure.

dashed curve to holes filled with silica (silica rods) and the dotted curve corresponds to air holes with a thin layer of silica on the hole walls (the thickness of the silica wall layer is $0.15 \Lambda$ ). In the following these $\mathrm{PhCs}$ are referred to as air-hole, silica-rod and silica-wall structures, respectively. Clearly, the largest bandgap is obtained when using air holes. However, the silica-rod structure and the silica-wall structure also show significant bandgaps. By

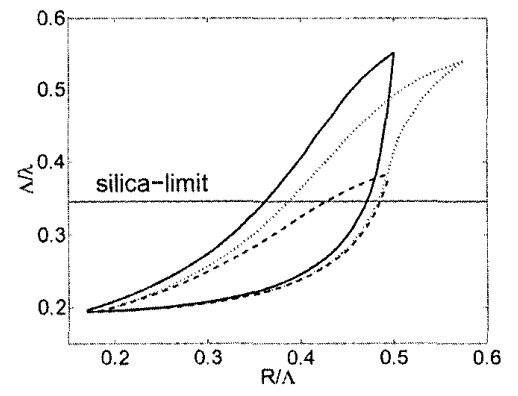

Figure 2: TE photonic bandgaps for silicon photonic crystal structures. Solid curve: air holes. Dashed curve: holes filled with silica (silica rods). Dotted curve: holes partially filled with silica (silica walls).

comparing two-dimensional calculations for infinite height crystals to the dispersion relation for silica, our substrate material, one finds that there is a fundamental upper 
frequency limit for leakage-free guidance of light for the corresponding finite height waveguide $/ 5 /$. For frequencies above this limit, shown in Fig. 2 as the silica-limit, light will leak into the silica substrate.

Fig. 2 shows that it is possible to obtain a reasonably large bandgap below the silica limit for TE polarized light for $\mathrm{PhC}$ structures with holes that are filled or partially filled with silica. The silica-rod structure has a much smaller index step than the air-hole structure and scattering at the interface will therefore be lower. For the silica-wall structure the index step will be softened and this will possibly also lead to reduced scattering.

We have fabricated PhCs with silica walls in the holes. A reasonable choice of air-hole radius in this case is $R=0.38 \Lambda$ for which a reasonably large bandgap exists below the silica-limit. An operating frequency near the centre of the bandgap is in this case $\Lambda / \lambda=0.28$, and for the optical wavelength $\lambda=1550 \mathrm{~nm}$ this results in the lattice constant $\Lambda=420 \mathrm{~nm}$ and the hole radius $\mathrm{R}=160 \mathrm{~nm}$. The height of the silicon slab layer is chosen to be $\sim 250 \mathrm{~nm}$ based on the 3D calculations in $/ 6 /$.

\section{Fabrication}

E-beam lithography was used to generate photonic crystal waveguide patterns on top of an SOI wafer (340nm silicon on $1000 \mathrm{~nm}$ silica). Holes were then etched through silicon and silica using reactive ion etching (RIE). Finally, silicon was thermally oxidized to grow a top cladding layer and a glass layer on the walls of the air holes. The thickness of the thermally grown silica was $-65 \mathrm{~nm}$. In addition to the softening of the index step, this glass layer might act to reduce scattering losses for another and probably more important reason: during oxidation the surfaces of the silicon slab layer (incl. the surface of the hole walls and the top surface) will become less rough.

Due to instabilities in our E-beam writing system the size of our patterns is presently limited to $40 \mu \mathrm{m}$ by $40 \mu \mathrm{m}$ or smaller. In order to be able to make transmission studies of waveguides defined within these small areas, we have designed a $90^{\circ}$ bend. The $90^{\circ}$ bend, which is shown in Fig.3, enables us to access the waveguides from two perpendicular facets (perpendicular facets are most easily obtained when cleaving silicon). The design of the bend is purely intuitive and is obtained by gradual rotation of the crystal lattice.

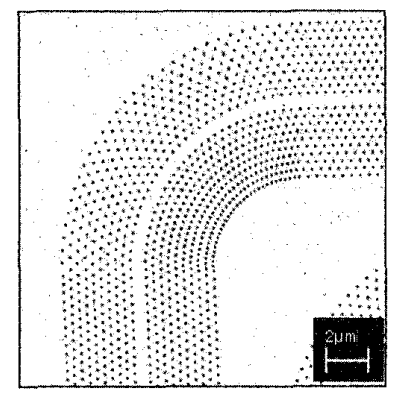

Figure 3: SEM micrograph of $90^{\circ}$-bended photonic crystal waveguide.

On our first sample with $90^{\circ}$-bended $\mathrm{PhC}$ waveguides for transmission measurements we had also defined $250 \mu \mathrm{m}$ long tapered ridge waveguides leading light to the photonic crystal area. The ridge waveguides served to relax the requirements to precision in cleaving. Unfortunately, the alignment between $\mathrm{PhC}$ waveguides and ridge waveguides was very poor on the sample.

\section{Optical characterization}

The transmission measurements showed a total loss (from input fibre to output fibre) of $\sim 40 \mathrm{~dB}$. This figure is not high considering the poor fibre-to-sample coupling (expected to account for at least $20 \mathrm{~dB}$ coupling loss) and the poor alignment mentioned above.

We also studied guiding of light in the same sample by looking at the sample from above with an infrared (IR) camera. Due to scattering at the points of poor alignment, we were able to follow clearly the path of guided light. One of the pictures is shown in Fig.4. From the IR pictures we were able to conclude that the $\mathrm{PhC}$ waveguides efficiently transmit light from the input ridge waveguide to the output ridge waveguide. We therefore estimate that the propagation losses in the bended $\mathrm{PhC}$ waveguides are quite small. In the near future, we expect to be able to determine values for the exact propagation and bending losses.

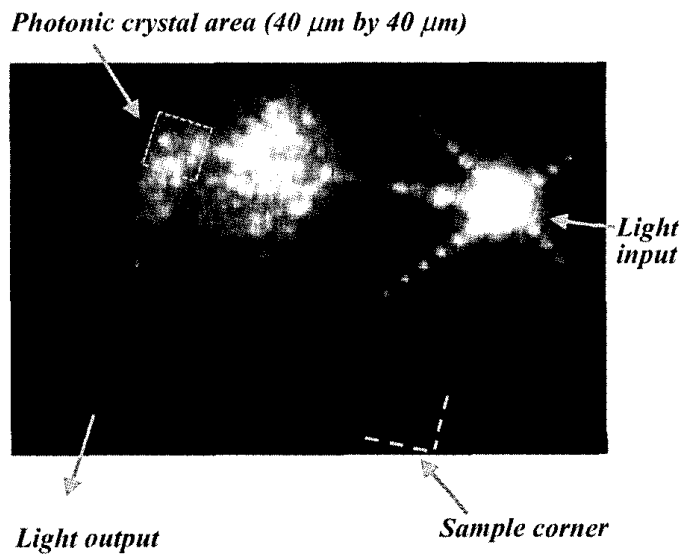

Figure 4: IR top view of photonic crystal waveguide sample.

In conclusion, we have realized robust silicon-on-insulator based photonic crystal waveguides. The structures are designed to be environmentally robust and to reduce outof-plane scattering. Transmission measurements and IR top view pictures indicate efficient guiding.

\section{References}

11/ T.F. Krauss et al., Prog. in Quantum Electron. 23 (1999) 51-96.

12/ M. Loncar et al., Appl. Phys. Lett. 77 (2000) $1937-$ 1939.

13/ C. Weisbuch et al., Synthetic Metals 116 (2001) 449 452.

14/ Ph. Lalanne et al., J. Appl. Phys. 89 (2001) 1512-14.

15/ T. Søndergaard et al., Appl. Phys. Lett. 77 (2000) 785-787.

16/ S.G. Johnson et al., Phys. Rev.B 60 (1999) 5751-58. 